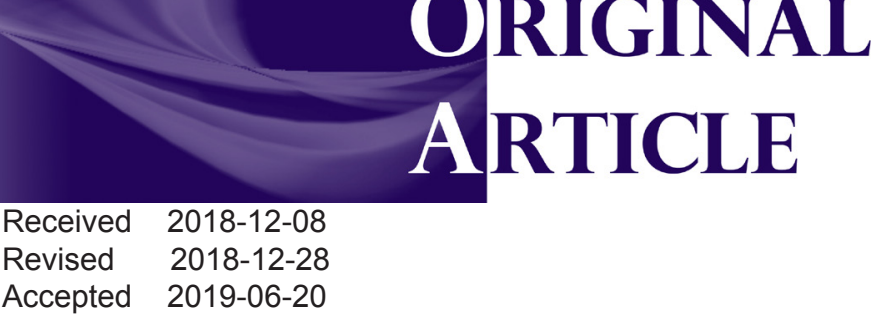

\title{
ORIGINAL
}

GMJ.2020;9:e1443

www.gmj.ir

$2018-12-28$

Accepted 2019-06-20

\section{Value of Transverse Groove on the Earlobe and Hair Growth on the Ear to Predict the Risk for Coronary Artery Disease and Its Severity among Iranian Population, in Tehran City}

\author{
Reza Arefi ${ }^{1}$, Mohammad Hassan Namazi ${ }^{2}$, Morteza Safi ${ }^{2}$, Habiboulah Saadat ${ }^{2}$, Hossein Vakili ${ }^{2}$, Mehdi Pishgahi ${ }^{2}$ \\ , Saeed Alipour Parsa $2 \bowtie$
}

Research Committee of AJA University of Medical Science, Tehran, Iran

${ }^{2}$ Shahid Beheshti University of Medical Sciences, Cardiovascular Research Center, Tehran, Iran

\begin{abstract}
Background: The use of phenotypic parameters along with other noninvasive diagnostic modality can lead to early diagnosis of coronary artery disease (CAD) and prevent its life-threatening outcome. Recently, the application of head and face components for assessing the risk for CAD much attention has been paid. The present study aimed to assess the relationship between ear characteristics (transverse groove on the earlobe and hair growth on the ear) and the risk for CAD and its severity among Iranian patients. Materials and Methods: In this cross-sectional study, the study population consisted of 105 consecutive patients with suspected CAD undergoing coronary angiography. The severity of CAD was determined by the number of disease vessels as well as the presence of left main lesions assessed by coronary angiography. All patients were examined to evaluate the appearance of ear regarding the presence of transverse groove on the earlobe and hair growth on the ear. Results: Comparing cardiovascular parameters across the groups with and without transverse groove on the earlobe showed a higher rate of CAD as well as the higher number of involved coronary arteries than in the groups without transverse groove on the earlobe. Similarly, the presence of CAD and its higher severity were more revealed in patients with hair growth on the ear as compared to the group without this phenotype. According to multivariable logistic regression analysis and with the presence of baseline parameters, the presence of transverse groove on the earlobe and hair growth on the ear increased the risk for CAD by 2.4 and 4.4 fold, respectively. Conclusion: Along with classic cardiovascular risk factors, the role of growing hair on the ear and transverse groove on the ear to predict high risk for CAD should be considered. [GMJ.2020;9:e1443] DOI:10.31661/gmi.v9i0.1443
\end{abstract}

Keywords: Groove; Earlobe; Hair Growth; Coronary Artery Disease; Iranian Population

GMJ

Copyright $\odot$ 2020, Galen Medical Journal. This is an open-access article distributed under the terms of the Creative Commons Attribution 4.0 Internation License (http://creativecommons.org/licenses/by/4.0/) Email:info@gmj.ir

\footnotetext{
Correspondence to:

Saeed Alipour Parsa, Shahid Beheshti University of Medical Sciences, Cardiovascular Research Center, Tehran, Iran

Telephone Number: +989121180516

Email Address: saeedalip@gmail.com
} 


\section{Introduction}

Coronary artery disease (CAD) is one of the major leading cause of mortality and morbidity, and even the second cause of death in most countries [1]. It has been estimated that by 2030 about one-third of the population in developed countries died or become disabled due to the progression of cardiovascular disorders and thus most of the healthcare costs will be allocated to the control and treatment of these diseases $[2,3]$. Till now, several planned approaches have been presented to predict the likelihood of significant CAD in suspected patients, especially in those with potential risk factors. Most of these algorithms are based on both clinical findings and imaging evidence achieving by invasive or semi-invasive modalities such as coronary angiography, perfusion scanning, and computed tomographic angiography $[4,5]$. Also, some specific limitations of such techniques due to their invasive nature or requiring radiation or contrasts agents, appearing complications following application of these procedures are not unexpected $[6,7]$. In this regard, the attention is focused on the simple naturally body markers for rapid predicting CAD risk. The earlobe characteristics, a simple clinical sign first described by Frank in 1973 as a good variable for predicting the risk of CAD [8]. Since then, some researchers have attempted to discover the pathogenic basis for the close link between parameters of earlobe and risk for CAD. Some studies described the close relationship between earlobe crease and increased risk for metabolic syndrome [9]. Also, some studies suggested a link between inflammatory markers such as macrophage activity and maintaining ear lobe collagen and loss of elastic fibers [10, 11]. However, the relationship between earlobe parameters and $\mathrm{CAD}$ remains uncertain with a meaningful relationship in studies and the lack of relation in another study [12]. More importantly, this association is highly affected by racial features and genetic tendencies. Hence, the present study aimed to assess the relationship between ear characteristics (transverse groove on the earlobe and hair growth on the ear) and the risk for CAD and its severity among Iranian population.

\section{Materials and Methods}

\section{Patients}

In this cross-sectional study, the population consisted of 105 consecutive patients with suspected CAD undergoing coronary angiography at Imam Reza and Shahid Modaresi hospitals in Tehran, Iran, during 2017-2018. All patients underwent coronary angiography according to the standard protocol. Definition of CAD was based on more than $50 \%$ reduction in luminal diameter of the major coronary artery, or one of its major branches.

\section{Sample Size Calculation}

According to the findings of previous studies, to reach the objectives of our study, the sample size was calculated to be 105 patients [13].

\section{Inclusion and Exclusion Criteria}

The patients with external ear disease or deformation, ear injury history, skin disease affecting ears, previous diagnosis of CAD and/or ischemic stroke, myocardial infarction, cardiomyopathy, and chronic diseases (such as cancer), were excluded from this study.

\section{Data Collection}

The severity of CAD was determined by the number of disease vessels as well as the presence of left main lesions. Baseline characteristics including gender, age, patients' weight and height, classic cardiovascular risk factors (e.g., hypertension, diabetes mellitus, smoking, alcohol consumption, and opium use) were collected by reviewing the hospital recorded files and entered into the study checklist. Along with reviewing the hospital files, all patients were examined for assessing the appearance of ear regarding the presence of transverse groove on the earlobe and hair growth on the ear. The study endpoint was to determine the relationship between ear characteristics and the presence and severity of CAD.

\section{Ethical Statements}

The study protocol was approved by the Ethics Committees at Iran University of Medical Sciences (approval number: Ir.SBMU.msp. rec.1396.651). 


\section{Statistical Analysis}

Descriptive analysis was used to describe the data, including mean \pm standard deviation (SD) for quantitative variables and frequency (percentage) for categorical variables. Chi-square test, independent t-test, and Mann-Whitney U test were used for comparison of variables. To determine the value of the transverse groove on the earlobe and hair growth on the ear to predict the presence of $\mathrm{CAD}$, the multivariable logistic regression analysis was employed. For the statistical analysis, the SPSS Statistics for Windows version 22.0 (IBM Corp. Released 2013, Armonk, New York, USA) was used. P-values $<0.05$ were considered statistically significant.

\section{Results}

The mean age of participants was $58.45 \pm$ 13.46 years (ranged $30-91$ years), and the mean body mass index was $26.23 \pm 2.66 \mathrm{~kg} /$ $\mathrm{m}^{2}$. Regarding occupational status, $59.0 \%$ had an army-dependent occupation status, $26.7 \%$ were employed, $13.3 \%$ were self-employed, and others were unemployed. Concerning classic cardiovascular risk factors, $40.0 \%$ were a current or former smoker, $20.0 \%$ were opium user, and $6.7 \%$ were alcohol user. Also, $24.7 \%$ and $39.0 \%$ have diabetes mellitus and hypertension, respectively. According to the coronary angiography reports, $19.0 \%$ of participants had normal coronary condition, while minimal CAD was revealed in $22.9 \%$, single-vessel disease in $15.2 \%$, two-vessel disease in $21.0 \%$, three-vessel disease in $17.1 \%$, and left main lesion in $4.8 \%$. The mean left ventricular ejection was $44.55 \pm$ $11.09 \%$ in which $23.8 \%$ had ejection fraction lower than or equal to $35 \%$. Comparison of baseline variables between the patients with and without transverse groove on the earlobe (Table-1) showed that the former group was significantly older and more hypertensive that as compared to those without this phenotype. Also, as shown in Table-2, those patients with hair growth on the ear were older, used alcohol more, and also suffered more from hypertension as compared to patients without hair growth on the ear. Comparing cardiovascular parameters across the groups with and without transverse groove on the earlobe showed a higher rate of CAD as well as the higher number of involved coronary arteries than in the groups without transverse groove on the earlobe (Table-3). Similarly, the presence of $\mathrm{CAD}$ and its higher severity were more revealed in patients with hair growth on the ear as compared to the group without this phenotype (Table-4). According to multivariable logistic regression analysis (Table-5) and with the presence of baseline parameters, the presence of transverse groove on the earlobe and hair growth on the ear increased the risk for CAD by 2.4 and 4.4 fold, respectively.

\section{Discussion}

The use of phenotypic parameters along with other noninvasive diagnostic modality can lead to early diagnosis of CAD and to prevent its life-threatening outcome. Recently, the application of head and face components for assessing the risk for CAD much attention has been paid $[12,13]$. Even, the use of these parameters in new risk cardiovascular scoring systems has been highly recommended [14]. In this regard, some indicators such as shape or size of facial components have been indicated to have special role in the suggested scoring systems [11, 12, 15]. In line with previous studies on the association between ear characteristics and the risk for $\mathrm{CAD}$, we examined the association between two parameters of the ear appearance including transverse groove on the ear and hair growth on the ear and presence of CAD [15]. We show that two pointed indicators could potentially predict CAD risk in suspected patients and candidate for coronary angiography. More interestingly, the predicting role of these two parameters is completely independent to other baseline CAD traditional risk profile. Till now, several cardiovascular risks scoring systems have been introduced such as the Framingham Coronary Heart Disease Risk Score [16], American College of Cardiology/American Heart Association (ACC/AHA) Risk score [17], the PREDICT-CVD secondary prevention score [18], atherosclerotic cardiovascular disease (ASCVD) [19], and WHO risk charts [20]. However, none of these scoring systems include apparent parameters. Regarding our findings, it can be strongly recommended to 
Table 1. Comparing Baseline Parameters between the Groups with and without Groove on the Ear Lobe

\begin{tabular}{cccc}
\hline Variables & $\begin{array}{c}\text { With groove } \\
(\mathbf{n = 6 6 )}\end{array}$ & $\begin{array}{c}\text { Without groove } \\
(\mathbf{n = 3 9 )}\end{array}$ & P-value \\
\hline Age, year & $61.88 \pm 11.81$ & $52.64 \pm 14.23$ & 0.001 \\
Body mass index, kg/m² & $26.26 \pm 2.68$ & $26.17 \pm 2.64$ & 0.871 \\
Occupational status & & & \\
Army-related & $41(62.1)$ & $21(53.8)$ & \\
Employed & $17(25.8)$ & $11(28.2)$ & 0.589 \\
Self-employed & $7(10.6)$ & $7(17.9)$ & \\
Unemployed & $1(1.5)$ & $0(0.0)$ & 0.410 \\
Smoking & $26(39.4)$ & $16(41.0)$ & 0.363 \\
Opium use & $15(22.7)$ & $6(15.4)$ & 0.627 \\
Alcohol consumption & $5(7.6)$ & $2(5.1)$ & 0.003 \\
Hypertension & $33(50.0)$ & $8(20.5)$ & 0.162 \\
Diabetes mellitus & $20(30.3)$ & $7(17.9)$ &
\end{tabular}

Table 2. Comparing Baseline Parameters between the Groups with and without Hair Growth on the Ear

\begin{tabular}{cccc}
\hline Variables & $\begin{array}{c}\text { With ear hair } \\
(\mathbf{n}=\mathbf{5 4})\end{array}$ & $\begin{array}{c}\text { Without ear hair } \\
(\mathbf{n = 5 1 )}\end{array}$ & P-value \\
\hline Age, year & $61.96 \pm 12.48$ & $52.26 \pm 13.02$ & $<0.001$ \\
Body mass index, kg/m $\mathbf{m}^{\mathbf{2}}$ & $26.16 \pm 2.76$ & $26.34 \pm 2.51$ & 0.736 \\
Occupational status & & & \\
Army-related & $44(65.7)$ & $18(47.4)$ & 0.224 \\
Employed & $15(22.4)$ & $13(34.2)$ & \\
Self-employed & $7(10.4)$ & $7(18.4)$ & 0.743 \\
Unemployed & $1(1.5)$ & $0(0.0)$ & 0.839 \\
Smoking & $27(40.3)$ & $15(39.5)$ & 0.039 \\
Opium use & $13(19.4)$ & $8(21.1)$ & 0.041 \\
Alcohol consumption & $7(10.4)$ & $0(0.0)$ & 0.198 \\
Hypertension & $31(46.3)$ & $10(26.3)$ & $7(18.4)$ \\
Diabetes mellitus & $20(29.9)$ & &
\end{tabular}

Table 3. Cardiovascular Status in the Groups with and without Groove on the Ear Lobe

\begin{tabular}{cccc}
\hline Variables & $\begin{array}{c}\text { With baldness } \\
(\mathbf{n = 5 4 )}\end{array}$ & $\begin{array}{c}\text { Without baldness } \\
(\mathbf{n}=\mathbf{5 1})\end{array}$ & P-value \\
\hline Presence of CAD & $57(86.4)$ & $28(71.8)$ & 0.036 \\
Severity of CAD & & & \\
None & $9(13.6)$ & $11(28.2)$ & \\
Minimal CAD & $11(16.7)$ & $13(33.3)$ & 0.007 \\
Single-vessel & $8(12.1)$ & $8(20.5)$ & \\
Two-vessel & $19(28.8)$ & $3(7.7)$ & \\
Three-vessel & $15(22.7)$ & $3(7.7)$ & 0.002 \\
Left main lesion & $4(6.1)$ & $1(2.6)$ & \\
\hline
\end{tabular}

CAD: Coronary Artery Disease 
Table 4. Cardiovascular Status in the Groups with and without Hair Growth on the Ear

\begin{tabular}{cccc}
\hline Variables & $\begin{array}{c}\text { With baldness } \\
(\mathbf{n = 5 4 )}\end{array}$ & $\begin{array}{c}\text { Without baldness } \\
(\mathbf{n = 5 1 )}\end{array}$ & P-value \\
\hline Presence of CAD & $60(89.6)$ & $25(65.8)$ & 0.003 \\
Severity of CAD & & & \\
None & $7(10.4)$ & $13(34.2)$ & \\
Minimal CAD & $16(23.9)$ & $8(21.1)$ & 0.042 \\
Single-vessel & $10(14.9)$ & $6(15.8)$ & \\
Two-vessel & $16(23.9)$ & $6(15.8)$ & \\
Three-vessel & $14(20.9)$ & $4(10.5)$ & 0.002 \\
Left main lesion & $4(6.0)$ & $1(2.6)$ & \\
\hline
\end{tabular}

Table 5. Multivariable Logistic Regression Modeling to Assess the Value of Transverse Groove on the Earlobe and Hair Growth on the Ear in Predicting CAD

\begin{tabular}{cccccc}
\hline Variable & B & S.E & Wald & OR & P-value \\
\hline Age & -0.061 & 0.032 & 3.664 & 0.940 & 0.056 \\
Body mass index & -0.231 & 0.135 & 2.920 & 0.794 & 0.087 \\
Smoking & 0.249 & 0.691 & 0.130 & 1.283 & 0.718 \\
Opium use & 1.175 & 1.018 & 1.330 & 3.237 & 0.249 \\
Alcohol use & 0.247 & 1.322 & 0.035 & 1.281 & 0.852 \\
Diabetes & 1.878 & 0.883 & 4.521 & 6.543 & 0.033 \\
Hypertension & 2.376 & 0.940 & 6.389 & 10.756 & 0.011 \\
Groove on the earlobe & -1.217 & 0.821 & 2.198 & 2.488 & 0.046 \\
Hair on the ear & -1.249 & 0.766 & 2.654 & 4.457 & 0.038 \\
Constant & -0.205 & 5.839 & 0.001 & 0.815 & 0.972 \\
\hline
\end{tabular}

add these parameters, especially two tested parameters in the present study to modify current scoring systems and achieve new systems with higher sensitivity and accuracy. Regarding the association between transverse groove on the ear and the risk for $\mathrm{CAD}$, reviewing the literature led to no similar studies. In other words, our study is the first survey on this association. Some studied focused on the relation between earlobe crease and increased risk for CAD. As indicated by Iorgoveanu et al. in 2018 [12], a close relationship could be suggested between the presence of the diagonal earlobe crease and CAD. A study by Honma et al. [14] in 2017, showed a close correlation between diagonal earlobe creases and coronary artery calcification score. In study by Aizawa et al. [15], the association between earlobe crease and left min coronary lesion has also been indicated. Regarding the pathogenesis of the increased likelihood of CAD in those with earlobe crease, some suggestions have been considered. Earlobe crease or Frank's sign was first described by Sanders T. Frank in 1973 [16], as a predictive dermatological finding of coronary artery disease. The detailed mechanisms of developing earlobe crease is uncertain, although it is speculated to be derived from loss or degeneration of elastic fibers by diminishing blood supply to the earlobes in a pathological study. Ziyrek et al. [21] showed an association between the presence of diagonal earlobe crease and increased carotid intima-media thickness and epicardial adipose tissue thickness that the latter indicator can own strongly predict the severity of coronary artery disease. It also seems that the role of earlobe crease in the prediction of CAD 
can be augmented by the presence of other CAD risk factors such as hypertension and diabetes [21]. As shown by Kamal in 2017 [13], the effect of hypertension and diabetes on the presence of diagonal earlobe crease is statistically significant. Concerning the association between the hair on the ear and risk for CAD, almost all studies focused on the relation between ear-canal hair and CAD risk. Thus, the significant association between CAD and hair on the ear can be the novel aspect of our study. Although several various factors [2225 ] can cause hair growth on the ear and may intervene (such as genetics, prostaglandins [E2 and F2 $\alpha$ ], growth hormone, insulin-like growth factor 1 , transforming growth factor beta 1 , tumor necrosis factor, basic fibroblast growth factor, thyroid hormones, glucocorticoids, estrogens, and androgens, etc.), based on the present study, growing hair on the ear (any point on auricle or earlobe) can powerfully predict the increased risk for CAD. The association between ear-canal hair and risk for CAD or acute myocardial infarction has also been pointed out in some previous studies [26, 27]. In an early study by Verma et al. [23] in 1989, a significant difference was also observed between men with and without CAD in the presence of ear-canal hair. Similar findings were revealed by Wagner et al. [28]. In a study by Ali et al. in 2010 [29], 37.2\% of CAD patients had hairy ear-canal, while the prevalence of other known risk factors for CAD such as hypertension or diabetes was lower. Also, they concluded that unknown risk factors for CAD such as hairy ear-canal might have a central role as compared to classic risk profile [29]. We also believed that the role of both ear-related indicators, including growing hair on the ear and transverse groove on the ear should not be ignored when modifying the scoring systems for predicting CAD and its severity.

\section{Conclusion}

Along with classic cardiovascular risk factors, the role of growing hair on the ear and transverse groove on the ear to predict high risk for CAD should be considered. Because of the pointed role for these indicators, considering such phenotypic components in modifying CAD risk scoring systems is quite logical.

\section{Acknowledgment}

Authors grateful to Parsian Teb Co. for their corrections pertaining to the English language.

\section{Conflict of Interest}

The authors declare no potential conflicts of interest.

\section{References}

1. Vasan RS, Benjamin EJ. The future of cardiovascular epidemiology. Circulation. 2016; 133(25): 2626-33.

2. Lee JT, Lawson KD, Wan Y, Majeed A, Morris S, Soljak M, et al. Are cardiovascular disease risk assessment and management programmes cost effective? A systematic review of the evidence. Prev Med. 2017; 99: 49-57.

3. Beyranvand MR, Piranfar MA, Mobini M, Pishgahi M. The relationship of st segment changes in lead avr with outcomes after myocardial infarction; A cross sectional study. Emerg. 2017; 5(1).

4. Nerlekar N, Ha FJ, Cheshire C, Rashid H, Cameron JD, Wong DT, et al. Computed tomographic coronary angiography-derived plaque characteristics predict major adverse cardiovascular events: A systematic review and meta-analysis. Circ Cardiovasc Imaging. 2018; 11(1): e006973.

5. Andelius L, Mortensen MB, Nørgaard BL, Abdulla J. Impact of statin therapy on coronary plaque burden and composition assessed by coronary computed tomographic angiography: a systematic review and metaanalysis. Eur Heart J Cardiovasc Imaging. 2018; 19(8): 850-858.

6. Kim C, Hong SJ, Shin DH, Kim JS, Kim BK, Ko YG, et al. Limitations of coronary computed tomographic angiography for delineating the lumen and vessel contours of coronary arteries in patients with stable angina. Eur Heart J Cardiovasc Imaging. 2015; 16(12): 
1358-65.

7. Kruk M, Wardziak Ł, Mintz GS, Achenbach S, Pręgowski J, Rużyłło W, et al. Accuracy of coronary computed tomography angiography vs intravascular ultrasound for evaluation of vessel area. J Cardiovasc Comput Tomogr. 2014; 8(2): 141-8

8. Frank ST. Aural sign of coronary-artery disease. N Engl J Med 1973; 289: 327-8.

9. Higuchi Y, Maeda T, Guan JZ, Oyama J, Sugano M, Makino N. Diagonal earlobe crease are associated with shorter telomere in male Japanese patients with metabolic syndrome. Circ J. 2009; 73: 274-9.

10. Sapira JD. Earlobe creases and macrophage receptors. South Med J 1991; 84: 537-8.

11. Shoenfeld Y, Mor R, Weinberger A, Avidor I, Pinkhas J. Diagonal ear lobe crease and coronary risk factors. J Am Geriatr Soc. 1980; 28: $184-7$

12. Iorgoveanu C, Zaghloul A, Desai A, Krishnan AM, Balakumaran K. Bilateral earlobe crease as a marker of premature coronary artery disease. Cureus. 2018; 10(5): e2616

13. Kamal R, Kausar K, Qavi AH, Minto MH, Ilyas F, Assad S, et al. Diagonal earlobe crease as a significant marker for coronary artery disease: A case-control study. Cureus. 2017; 9(2): e1013.

14. Honma M, Shibuya T, Iwasaki T, Iinuma S, Takahashi N, Kishibe M, et al. Prevalence of coronary artery calcification in Japanese patients with psoriasis: A close correlation with bilateral diagonal earlobe creases. J Dermatol. 2017; 44(10): 1122-1128.

15. Aizawa T, Shiomi H, Kitano K, Kimura T. Frank's sign: diagonal earlobe crease. Eur Heart J. 2018; 39(40): 3653.

16. Hoffmann U, Massaro JM, D'Agostino $\mathrm{Sr}$ RB, Kathiresan S, Fox CS, O'Donnell CJ. Cardiovascular event prediction and risk reclassification by coronary, aortic, and valvular calcification in the Framingham Heart Study. J Am Heart Assoc. 2016; 5(2): e003144.

17. Mosepele M, Hemphill LC, Palai T, Nkele I, Bennett K, Lockman S, et al. Cardiovascular disease risk prediction by the American College of Cardiology (ACC)/American Heart Association (AHA) Atherosclerotic Cardiovascular Disease (ASCVD) risk score among HIV-infected patients in sub-Saharan Africa. PloS One. 2017; 12(2): e0172897.

18. Poppe KK, Doughty RN, Wells S, Gentles D, Hemingway H, Jackson R, et al. Developing and validating a cardiovascular risk score for patients in the community with prior cardiovascular disease. Heart. 2017; 103(12): 891-2.
19. Budoff MJ, Young R, Burke G, Jeffrey Carr J, Detrano RC, Folsom AR, et al. Tenyear association of coronary artery calcium with atherosclerotic cardiovascular disease (ASCVD) events: the multi-ethnic study of atherosclerosis (MESA). Eur Heart J. 2018; 39(25): 2401-8.

20. Bansal P, Chaudhary A, Wander P, Satija M, Sharma S, Girdhar S, et al. Cardiovascular risk assessment using WHO/ISH risk prediction charts in a rural area of North India. J Res Med Dent Sci. 2016; 4(2): 127-31.

21. Ziyrek M, Şahin S, Özdemir E, Acar Z, Kahraman S. Diagonal earlobe crease associated with increased epicardial adipose tissue and carotid intima media thickness in subjects free of clinical cardiovascular disease. Turk Kardiyol Dern Ars. 2016; 44(6): 474-80.

22. Eroglu S, Sade LE, Yildirir A, Bal U, Ozbicer S, Ozgul AS, et al. Epicardial adipose tissue thickness by echocardiography is a marker for the presence and severity of coronary artery disease. Nutr Metab Cardiovasc Dis. 2009; 19(3): 211-7.

23. Verma SK, Khamesra R, Mehta LK, Bordia A. Ear-lobe crease and ear-canal hair as predictors of coronary artery disease in Indian population. Indian Heart J. 1989; 41(2): 86-91.

24. Oh HS, Smart RC. An estrogen receptor pathway regulates the telogen-anagen hair follicle transition and influences epidermal cell proliferation. Proc Natl Acad Sci U S A. 1996; 93(22): 12525-30.

25. Little JC, Redwood KL, Granger SP, Jenkins $\mathrm{G}$. In vivo cytokine and receptor gene expression during the rat hair growth cycle: Analysis by semi-quantitative RT-PCR. Exp Dermatol. 1996; 5(4): 202-12.

26. Agouridis AP, Elisaf MS, Nair DR, Mikhailidis DP. Ear lobe crease: a marker of coronary artery disease? Arch Med Sci. 2015; 11(6): 1145.

27. Kumar A. Frank's sign and coronary artery disease in Indian population. Heart India. 2016; 4(4): 129.

28. Wagner RF Jr, Reinfeld HB, Wagner KD, Gambino AT, Falco TA, Sokol JA, et al. Earcanal hair and the ear-lobe crease as predictors for coronary-artery disease. N Engl J Med. 1984; 311(20): 1317-8.

29. Ali RA, Asadollah M, Hossien RA. The role of unknown risk factors in myocardial infarction. Cardiol Res. 2010; 1(1): 15-19. 